\title{
Extended viewing angle holographic display system with tilted SLMs in a circular configuration
}

\author{
Tomasz Kozacki, ${ }^{1, *}$ Małgorzata Kujawińska, ${ }^{1}$ Grzegorz Finke, ${ }^{1}$ \\ Bryan Hennelly, ${ }^{2}$ and Nitesh Pandey ${ }^{2}$ \\ ${ }^{1}$ Institute of Micromechanics and Photonics, Warsaw University of Technology, 8 Sw. A. Boboli Street, 02-525 Warsaw, Poland \\ ${ }^{2}$ Department of Computer Science, National University of Ireland, Maynooth, Co. Kildare, Ireland \\ ${ }^{*}$ Corresponding author: t.kozacki@mchtr.pw.edu.pl
}

Received 9 December 2011; revised 15 February 2012; accepted 15 February 2012;

posted 17 February 2012 (Doc. ID 158845); published 6 April 2012

\begin{abstract}
This paper presents an extended viewing angle holographic display for reconstruction of real world objects in which the capture and display systems are decoupled. This is achieved by employing multiple tilted spatial light modulators (SLMs) arranged in a circular configuration. In order to prove the proper reconstruction and visual perception of holographic images the Wigner distribution function is employed. We describe both the capture system using a single static camera with a rotating object and a holographic display utilizing six tilted SLMs. The experimental results based on the reconstruction of computer generated and real world scenes are presented. The coherent noise removal procedure is described and implemented. The experiments prove the possibility to view images reconstructed in the display binocularly and with good quality. (C) 2012 Optical Society of America
\end{abstract}

OCIS codes: $\quad 090.2870,090.1995,090.1760$.

\section{Introduction}

The most futuristic vision of a device generating three-dimensional (3-D) images requires delivering a ghost-like, high quality optical replica of a moving object or scene that is floating in space and can be viewed by an observer freely from different angles. This vision is often referred as True 3-D [1]. This idea is a very different one from the recent developments in stereoscopic and autostereoscopic systems [2] where depth information of a generated view is not preserved. The most natural way to realize the postulate of True 3-D video is the application of holography [3]. Using holography a complex optical wave is created similar to that which is generated in the real world, and therefore, 3-D object wave fronts may be correctly reconstructed.

Holography in video application allows for imaging a natural copy of 3-D moving scenes. This is a very

1559-128X/12/111771-10\$15.00/0

(C) 2012 Optical Society of America distinct approach from the stereoscopic and autostereoscopic 3-D TV systems, which are based on a certain manipulation of two or more 2-D images [4]. Stereoscopic techniques, although easy for commercial implementation, are far from the vision of true optical replicas. They have several major drawbacks including: (1) the viewer's discomfort due to the physical duplication of 2-D light distributions that reach their eyes, and (2) the perceived image is independent from the observer position. The first disadvantage of stereoscopic systems is that the two depth cues, accommodation and perceived three-dimensionality, give contradictory information to the brain. For the accommodation cue the image is flat, while binocular vision (images mutual shift) gives a 3-D view. A second disadvantage is that there are only two views (or a small number of them), which provide a perceived three-dimensionality that is independent from the viewer position (or the dependence is small). For holographic displays both cues are realized naturally, and the number of views given by a holographic display is infinite (for a holographic display 
employing a HD modulator panel of $1920 \times$ 1080 pixels there are more than 2 megaFviews).

The constant development of charged coupled devices (CCDs) and spatial light modulator (SLM) technology in the last decades has given new possibilities of data recording and presentation and has contributed to form the digital holography based concepts of 3 -D displays [5,6]. Nowadays, for building such systems, devices based on liquid crystals are being recognized as the most suitable since they provide a very high accuracy in optical wave front reproduction $[7,8]$. However, their small size and relatively big pixels impose the utilization of more than one SLM in a holographic display system. To overcome this problem, in the literature several attempts have been made to enhance this feature by employing innovative optical holographic display modules with several tilled SLMs [9-12]. There are two major display approaches, based on a flat geometry and a circular one. The maximum bandwidth of a planar display depends on the pixel size only. Therefore for the purpose of extending the viewing angle the circular configuration is the preferable one. In the display proposed in this paper we present a system composed of several SLMs, arranged in a circular configuration. A solution like this allows for an increase in the viewing angle of the reconstructed image, which results in an increased horizontal parallax and improved viewing comfort.

In a recent publication we presented the concept of a multi CCD-multi SLM holographic capture and display system [13]. However, the design presented there has a number of problems: (1) the holographic reconstructions given in the system are accompanied with gaps; (2) the complexity of the system increases significantly when the number of SLMs increases, and (3) a change of the reconstruction distance is troublesome. The design problems were connected with the direct linking of the capture and the display systems. The recorded optical field is directly displayed on the SLM, but there is generally a pixel and wavelength mismatch between the capture and display systems. In this paper we present a display design where the capture and display systems are decoupled. The SLM arrangement is not a copy of CCD arrangement, and therefore, the captured holograms have to be processed prior to their display. Processing includes complex object wave retrieval from the captured intensity data followed by propagation of the object beam from the CCD conjugated plane to the SLM plane.

In Section 2 of the paper we employ the Wigner distribution function (WDF) [14] in order to present a theoretical analysis of a purely hypothetical holographic display with its pixels arranged circularly, where we focus on analyzing the holographic image that is reconstructed and on its visual perception. We show that the parameters describing such a system are equivalent to those representing a holographic display with circularly distributed planar SLMs. In Section 3 we present a holographic capture system with a rotating object that is used to capture data for our display system, while in Section 4 we present a holographic display design utilizing six tilted SLMs arranged on a circle. A very troublesome feature of holographic systems is the presence of coherent noise in holographic images. Therefore in the paper we present a technical solution that reduces the effect of this noise. This solution includes modifications in the holographic capture and display setups and it is discussed in Sections 3 and 4 . Finally in Section 5 we present the holographic images obtained using our holographic display system and analyze the possibility to view them binocularly.

\section{Display System Wigner Distribution Analysis}

A purely theoretical holographic display in a circular configuration (SLM with pixels that are distributed on a circle) is presented in Fig. 1. It will be shown that such a configuration well approximates the optical properties of a holographic display with multiple planar SLMs distributed on circle (i.e., the design proposed in the paper). We will therefore first analyze the holographic display with the SLM pixels distributed on a circle and then we present its approximation which is assembled from flat panels distributed on the circle.

To analyze the imaging properties of the circular display we employ phase space diagrams that illustrate the WDF. We assume that the paraxial approximation can be used to describe wave propagation. Under this approximation the WDF is mapped as follows: $W(x, f) \rightarrow W(x-\lambda z f, f)$ where $x$ and $f$ denote the space and spatial frequency coordinates, $\lambda$ is the wavelength of the light, and $z$ denotes the distance of wave propagation. We limit our analysis to 1-D only; extension to 2-D is trivial. The bounds of the WDF of the optical signal at the SLM plane $(z=0)$ and the image plane $(z=R)$ are shown in the left and right parts of Fig. 2(a), respectively.

The WDF shape for the SLM plane $(z=0)$ is vertically sheared due to the spherical nature of the SLM shape and the resulting inclination angle is given by $\lambda R^{-1}$. This is identical to the effect of a negative thin lens on the WDF shape. The tilted dotted line crossing in the middle of the WDF plot represents a spherical beam generated by the SLM replaying a signal of constant phase. Locally at any point of the SLM we can change the phase pattern displayed

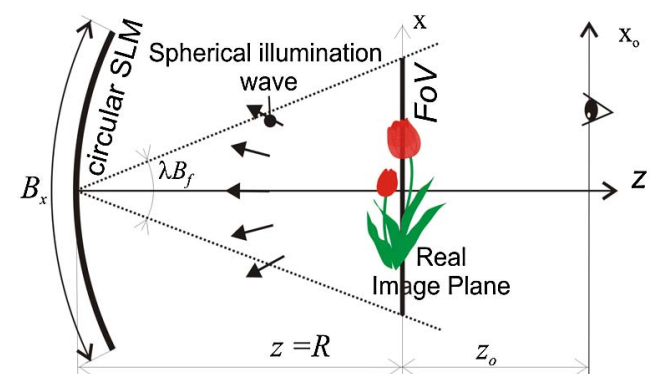

Fig. 1. (Color online) Holographic display system with theoretical circular SLM. 

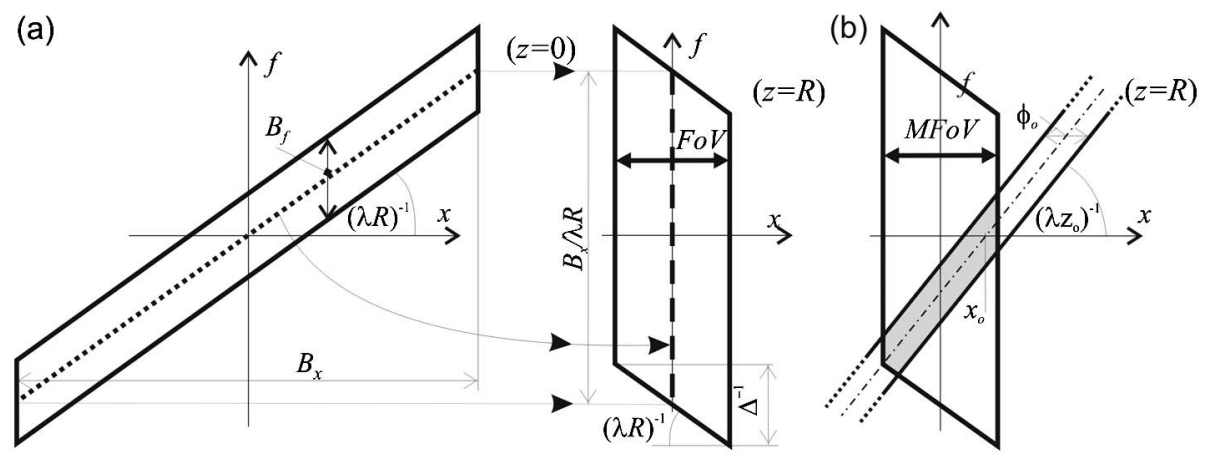

Fig. 2. (a) WDF representation of the optical signal generated at planes $z=0$ (left region) and $z=R$ (right region). (b) WDF representation of visual perception.

on the SLM with limits on the bandwidth of that pattern given by $\left(-\frac{1}{2} B_{f}, \frac{1}{2} B_{f}\right)$ relative to the spherical carrier wave. Focusing of the spherical carrier (i.e., propagation a distance $R$ ) is illustrated in Fig. 2(a) (right figure part) where the WDF is now horizontally sheared as a result of the Fresnel propagation. The dotted line in the WDF on the left (at $z=0$ ) is mapped to the dashed line in the WDF on the right $(Z=R)$. A vertical line in the phase space diagram represents a Dirac delta function in $x$. Thus the spherical wave is focused to a point under propagation by the distance $R$. In the circular configuration the entire SLM bandwidth is utilized and the WDF representation has a shape that is stretched along the frequency axis only. The full SLM bandwidth contributes to the formation of each image point in the reconstruction plane. In the case of a planar SLM the horizontal shearing of the WDF associated with propagation effectively reduces the local bandwidth of the resulting image. When we apply a linear phase distribution of some spatial frequency on the circular SLM, then at the reconstruction plane we obtain a point image of position determined by value of that spatial frequency. When we apply a linear phase of maximum (or minimum) SLM frequency we will get marginal point images at $x= \pm \mathrm{Fov} / 2\left(F_{O} V\right.$-linear field of view). In this paper we use the definition of linear $F_{O} V$, which is more convenient for the present discussion than the more traditional angular field of view. It is possible to determine the field of view as follows:

$$
F o V=\lambda R \Delta^{-1}
$$

where $\Delta$ is the sampling period of the SLM. This equation is equivalent to the $F_{O} V$ of planar displays [15]. However, there are two important distinctions to be made: for the circular display the resolution over the entire image $F_{O} V$ is constant: $B_{x} / \lambda R, B_{x}=$ $N \Delta$ and $N$ is a number of SLM samples. This is an interesting feature of holographic displays with a circular shape. The entire bandwidth of the SLM can be used in generating the holographic image. In a planar holographic display some of the bandwidth is lost due to aliasing effects. For the planar SLM case aliasing effects result in higher orders that slightly invade the field of view of the zero order while for the circular SLM this does not happen.

We now proceed to analyze the process of observing a holographic image in the circular holographic display geometry again using the WDF representation [Fig. 2(b)]. In order to analyze the effect of monocular perception we introduce an eye aperture with a pupil size $\varphi_{0}$ placed at location $\left[x_{o}, z_{o}\right]$. This aperture covers the portion of an optical signal that is captured by an eye and it represents the act of viewing of the reconstructed image. In Fig. 2(b) the WDF chart of an eye aperture and of the holographic image are shown for the reconstruction plane, the Wigner chart of an eye is found by backpropagating the WDF of a rectangular function of width $\phi_{0}$ to the image plane. The overlap represents the energy from an image that is actually captured by an eye and it is illustrated by the shaded area. The spatial size of this overlap defines the monocular $F_{O} V\left(M F_{O} V\right)$. In Fig. 2(b) we present the case in which $\mathrm{FoV}=\mathrm{MFoV}$ for some variation of the eye position. This is a desired feature of displays; the size of the viewed area shall not depend on the angle of perception. Therefore we first define the minimum observation distance where for axial observation $\mathrm{MFoV}=\mathrm{FoV}$ :

$$
z_{o \min }=\frac{\lambda R^{2}}{B_{x} \Delta-\lambda R}
$$

For observation distances $z_{o}<z_{o \text { min }}$ the size of the monocular view is smaller than $F_{O} V$ and the $M F_{O} V$ will move across the $F o V$ with a transverse change of an observation point. Therefore, we limit our discussion to $z_{o}>z_{o \text { min }}$. For such a viewing distance we characterize an observation area $\left(\left|x_{O}\right|<x_{\text {olim }}^{(\mathrm{MFoV}=\mathrm{FoV})}\right)$, where an eye can move and the viewer sees an image of the same size and position (constant $\mathrm{MFoV}=$ $\mathrm{FoV}$ ). If an eye moves beyond this range, the $M F_{O} V$ decreases linearly from the maximum value to zero width for $x_{o l i m}^{(\mathrm{MFoV}=\mathrm{FoV})}>\left|x_{O}\right|>x_{o \lim }^{(\mathrm{MFoV}=0)}$. Using the WDF graphical representation illustrated in Fig. 2(b) we can deduce the relation for $M F O V$ as a function of observer position $x_{o}$ is given as: 


$$
M F_{O} V=\left\{\begin{array}{cc}
F o V & \left|x_{O}\right|<x_{o \lim }^{(M F o V=F o V)}, \\
\frac{R}{z_{o}+R}\left(\frac{B_{x} z_{o}}{2 R}+\frac{\lambda\left(z_{o}+R\right)}{2 \Delta}-x_{o}\right) & x_{o \lim }^{(M F o V=F o V)}>\left|x_{O}\right|>x_{o \lim }^{(M F o V=0)} \\
0 & \text { otherwise }
\end{array}\right.
$$

where

$$
\begin{aligned}
& x_{o \lim }^{(M F V=F o V)}=\frac{B_{x} z_{o}}{2 R}-\frac{\lambda\left(z_{o}+R\right)}{2 \Delta}, \\
& x_{o \lim }^{(M F O=0)}=\frac{B_{x} z_{o}}{2 R}+\frac{\lambda}{2 \Delta}\left(z_{o}+R\right) .
\end{aligned}
$$

For binocular observation when we view holographic images the $M F o V$ for both left and right eyes will be equal to $F_{O} V$. This gives a condition for stereoscopic observation; we require that $x_{\text {olim }}^{(\mathrm{MFoV}=\mathrm{FoV})}=d_{b} / 2$, where $d_{b}$ is the interpupillary distance of the eyes. From this condition we can compute the minimum observation distance for which at axial observation for both eyes we satisfy the condition $\mathrm{FoV}=\mathrm{MFoV}$

$$
z_{b \lim }=\frac{d_{b} \Delta R+\lambda R^{2}}{B_{x} \Delta-\lambda R}
$$

In this paper we discuss a holographic display design that is assembled from reflective LCoS (Liquid Crystal on Silicon) SLMs. The design is illustrated in Fig. 3(a), where a set of six parallel beams illuminates a set of six planar SLMs aligned on the circle. The beam reflected from the SLMs points toward a circular alignment focal point. An SLM has a mounting component around the active area. Therefore the active areas of adjacent SLMs are not coinciding and our pixel distribution contains gaps. Fig. 3(b) illustrates a WDF representation of the bounds within the holographic image. The dotted lines indicate the WDF of the circular display that was previously discussed and the gray regions represent the signal
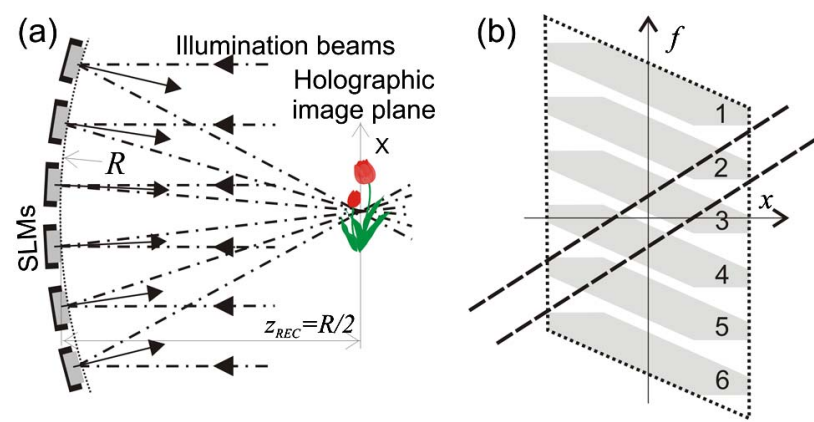

Fig. 3. (Color online) (a) Illustration of the holographic display system with planar SLMs aligned on a circle. (b) WDF representation of the optical signal generated at plane $z=R$ in a display, the dotted line illustrates the WDF of the circular display and the gray regions illustrate the WDF of the display built from planar SLMs. The larger dashed line illustrates the WDF of an eye aperture that is backpropagated to the image plane. that can be created by the planar SLMs. As we can see from the figure, the practical display with planar SLMs well approximates the theoretical circular display. Discrepancies are caused by the gaps and also by the SLMs flatness [13]. Gaps in the WDF representation result in regions of constant width in the frequency domain, a change of the separation between SLMs on the circle results in a change of the width of frequency gap [13]. Triangular white regions at the $F_{O} V$ limits are caused by the flatness of the SLMs [15].

We now consider visual perception where the WDF bounds of an eye is represented with dashed lines [Fig. 3(b)]. The effect of the gaps can be found by analyzing projections of the common area of the eye aperture WDF bounds and the WDF of the holographic image on the $x$ axis. Then the gaps in circular arrangement result in a modulation of the intensity and resolution of the obtained views. We refer to this effect as a modulation function of the reconstructed view (MFRV). The function $M F R V$ can be found by integration of the WDF representation of the visual perception with respect to the frequency:

$$
\begin{aligned}
\operatorname{MFRV}\left(x_{o}^{\prime}\right)= & \int \sum_{n=-3}^{2} \Pi\left(\frac{\lambda z}{B_{x}} f-\mathrm{FF}^{-1}\left(n+\frac{1}{2}\right)\right) \\
& \times \Pi\left(\frac{\lambda z_{o}}{\phi_{o}} f-\frac{x_{o}^{\prime}}{\phi_{o}}\right) \mathrm{d} f,
\end{aligned}
$$

where we introduce $x_{o}^{\prime}=x_{o}-x$ and a fill factor parameter (FF). The integral can be recognized and computed as a convolution. To quantify the effect of gaps we compute the contrast of the $M F R V$ function:

$$
C\{\mathrm{MFRV}\}=\frac{B_{x} z^{-1}\left(\mathrm{FF}^{-1}-1\right)}{2 \phi_{o} z_{o}^{-1}-B_{x} z^{-1}\left(\mathrm{FF}^{-1}-1\right)} .
$$

In our experimental configuration of the display the SLM active area has a width of $15.36 \mathrm{~mm}$ and the SLM housing has a width of $24 \mathrm{~mm}$ resulting in a maximum value of the $\mathrm{FF}=0.64$. In Section 5 we present the experimental holographic display configured for a reconstruction distance of $z=700 \mathrm{~mm}$. For this case an observation distance of $z_{o}=500 \mathrm{~mm}$ and an eye aperture size of $\phi_{o}=8 \mathrm{~mm}$ the contrast 
$C\{\mathrm{MVRV}\}$ equals 0.63 . For the same observation condition and $\mathrm{FF}=0.57$ contrast function $C\{\mathrm{MVRV}\}$ gets maximum value of 1 . For $F F$ smaller than 0.57 the observed image will start to have blank areas that are absent of image data. We note that the analysis above is valid in the central area of the FoV of the holographic image, i.e., in an area of size given by FoV $-B_{x}$. We note that for SLMs that are tilted with respect to the illumination beam, both the resolution and the $F_{O} V$ changes [16], however the effect can be neglected since in our display the tilt is small.

\section{Capture System}

In this section we report on the procedures used for the recording of static case phase shifting digital holograms (PSDH) [17], used as input to the circular display discussed in the previous section. Using the phase shifting technique we can record high quality digital holograms that are effectively free of the noise due to the DC and twin images terms. The disadvantage of the PS recording technique is that it requires four sequential captures, and therefore, it is not practical for recording real time moving scenes. However, moving videos can be created using piecewise stop motion of the objects. Furthermore, we can take advantage of the fact that the object is not moving in order to record holograms with multiple different diffuser illumination for speckle reduction. Below we describe the recording setup, our reasons for using PSDH, and the recording of stop motion videos of rotating objects with speckle reduction.

\section{A. Recording Setup}

In Fig. 4 we show the setup used to record digital holograms for our display system. We use a laser of power $100 \mathrm{~mW}$ and wavelength $785 \mathrm{~nm}$. The laser passes through a neutral density filter which allows us to vary the input power to the system as required. A linear polarizer and a quarter wave plate follow next. By varying these two elements we can control the relative power that is output from the two arms of the polarizing beam splitter which is the next element to be encountered. The horizontal arm of the

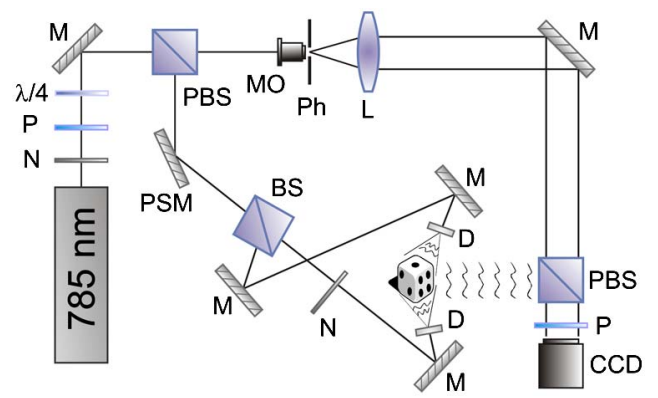

Fig. 4. (Color online) Illustration of phase shifting digital holograms recording setup. N: Neutral Density Filter, P: Polarizer, $\lambda / 4$ : Quarter Wave Plate, MO: Microscope Objective, PBS: Polarizing Beam Splitter, BS: Beam Splitter, Ph: Pinhole, L: Lens, M: Mirror, PSM: Phase Shifting Mirror, D: Diffuser. beam splitter travels through a microscope objective that converges the light. The light passes through a pinhole with a $10 \mu \mathrm{m}$ diameter which spatially filters the light and it then passes through a lens which collimates the light and creates a plane wave reference beam which propagates directly toward the CCD camera $(1920 \times 1080$ pixel Basler with a pixel pitch of $7.4 \mu \mathrm{m}$ and GigE interface) via a mirror, another polarizing beam splitter, and a polarizer.

The second arm of the first beam splitter is reflected from a mirror attached to a piezoelectric motor which can be moved by fractions of a wavelength for PSDH. This 'object' beam is then passed through a beam splitter which allows us to create two object beams so objects from two directions can be illuminated to ensure shadows are avoided. Both beams are directed towards opposite sides of the object via a system of mirrors and they are both passed through diffusers which spread the light out to illuminate the entire object. The light is scattered directly toward the final beam splitter which reflects the light toward the camera. Both the reference and object wave fields passes through a linear polarizer just before they reach the camera. This linear polarizer forces both wave fields into the same state of polarization so that an interference pattern may be recorded. There is no pinhole in the object arm as we seek to maximize the power of the light illuminating the object. Also the object arm and reference arm are approximately matched in optical path length maximizing the temporal coherence of the system and the quality of the digital holograms that are recorded.

\section{Recording of Stop Motion Videos with Speckle Reduction}

In our setup an object is positioned on a rotation stage and the diffusers in the setup described above are moved using a translation stage. An integrated lab view control system was designed for the entire setup so stop motion videos of rotating objects could be recorded (with multiple different diffuser illumination positions for each object position.

Speckle noise is a problem in all forms of coherent imaging [18]. In order to reduce the speckle in our display we propose to illuminate the object with a moving diffuser. The diffuser is moved between PSDH recordings. In this way for a given object position we can record multiple holograms which when reconstructed will contain statistically independent speckle noise patterns. To reduce this noise we can average the intensities of these reconstructions. This can be achieved numerically by simply adding the intensities of the numerical reconstructions, or optically by displaying these holograms at a rate that is fast enough to average within the integration time of the human eye.

The higher the number of diffuser positions that we select in the recording process, the better the speckle reduction that can be achieved. This is assuming that for each object position all of these digital holograms could be displayed in sequence 


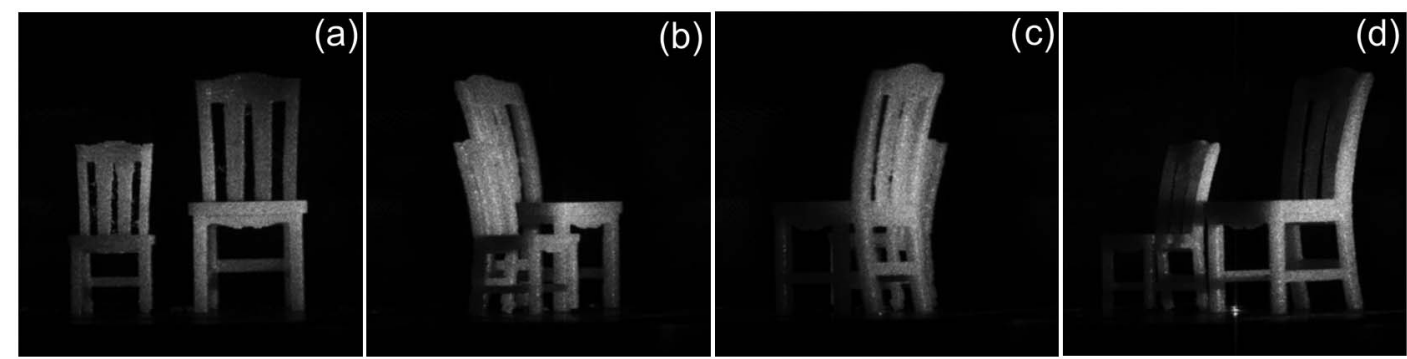

Fig. 5. Numerical reconstructions of holograms of two miniature chairs as seen from different perspectives. The speckle reduction is achieved by adding intensities of four reconstructions.

sufficiently quickly to integrate in the integration time of the human eye. The maximum frame rate of the LCOS SLMs is $60 \mathrm{~Hz}$. In our display we have decided to the limit display frequency to $15 \mathrm{~Hz}$ and use four frames for speckle reduction. This frequency choice gives reasonable noise reduction and acceptable flickering effect. In general, there are faster SLMs on the market so they can deliver a faster display frequency, and therefore, a greater reduction of speckle. For this reason we record only four PS digital holograms with different diffuser positions for each object position for all our videos. With this we can achieve approximately $50 \%$ speckle contrast reduction without losses in resolution or angular perspective. We note that if the sampling rate of the SLMs were to increase we could record more diffuser positions and achieve greater speckle reduction. In theory if it is possible to display $N$ images of the same objects (but having a different diffuse illumination on the recording side for each frame) within the integration time of the eye, it is possible to reduce the speckle contrast by $1 / N^{0.5}$. For example, we have demonstrated this using 10 such digital holograms added together over the integration time of a camera [8]. The difficulty in achieving the summation of a large number of speckle patterns lies in both the recording and display of high speed scenes using cameras and SLMs with fast rates. Furthermore, we also note that this approach cannot be taken for the single shot digital holograms of moving objects unless the object motion was sufficiently slow with respect to the capture/display rate, that is, could be assumed to be static.

In Fig. $\underline{5}$, we show the numerical reconstruction obtained from videos of PSDH recorded using the optical setup and software discussed above. We show four reconstructions from angles separated by approximately 60 degrees. Here the reconstructions were calculated numerically using the direct method of simulating the Fresnel propagation [19], using a single FFT algorithm. The intensity of the numerical reconstruction is calculated and the four intensities corresponding to the four different diffuser positions are added together in order to simulate what the eye will see. The two chairs were recorded at a distance of $436 \mathrm{~mm}$ relatively to the CCD and with an angular step of 1.58 degrees. In total 228 different object positions were recorded.

\section{Display System}

The design of the holographic display is based on the idea presented in Fig. 3(a), where six SLMs, illuminated with parallel beams are distributed on a circular segment. A detailed optical scheme and system photos are shown in Fig. 6(a) and 6(b), respectively. We use a Holoeye 1080P liquid crystal on silicon (LCOS) phase only SLM with the parameters: $N_{x} \times$ $N_{y}(1920 \times 1080)$ and pixel pitch $8 \mu \mathrm{m}$. The light source is the Nd-YAG laser with $\lambda=532 \mathrm{~nm}$. The illumination beams are linearly polarized along the LC molecules orientation direction, and therefore, the phases of these beams are modulated only. The display system consists of two modules: illumination and reconstruction. The task of the first one is to illuminate six SLMs with homogeneous, parallel beams with the same intensity. For this purpose we use a single laser beam, where a plane wave is obtained by a large diameter collimating lens. This beam is divided into six parts by means of three beam-splitter cubes. The SLMs are illuminated by vertically tilted beams using a set of independent mirrors. The tilt is necessary to separate incident and reflected beams, however the vertical tilt is small (approximately $1.5^{\circ}-2^{\circ}$ ), so the negative effects of it may be neglected. In our system the multiple SLMs are placed laterally, and therefore there is relatively small space-bandwidth product in the vertical direction. In the system an asymmetric diffuser is placed in the center of the real image reconstruction plane as shown in Fig. 6 . The diffuser scatters light mainly in one direction only $(y)$. The asymmetric diffuser plays a role of a medium which at the cost of removing vertical parallax extends the size of the observable image. This allows for a more convenient holographic image observation i.e., the eye may take a wide range of positions in the $y$ direction. The scattering angles of the diffuser are $0.2^{\circ} \times 40^{\circ}$. We note that the angular perspective of the holographic image in the $x$ direction is only weakly affected. The $x$ directional scattering limits the perceived threedimensionality in the $z$ direction. We have experimentally quantified the effect of the diffuser for a reconstruction distance of $700 \mathrm{~mm}$ and we have found that it limits the reconstruction to images of $z$ direction extent approximately equal to $60 \mathrm{~mm}$.

Previously, we have reported on a holographic display system where the SLMs are all orientated 

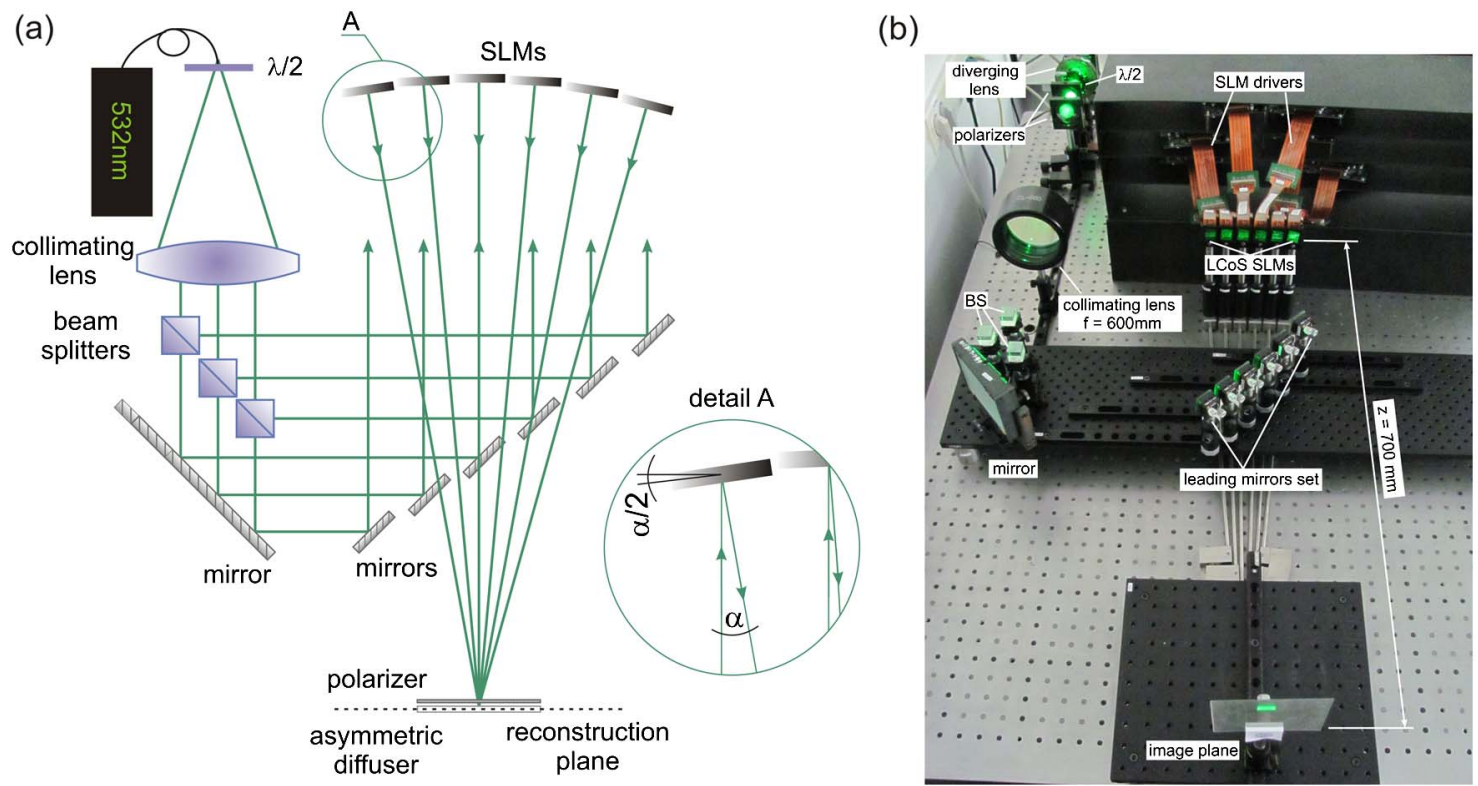

Fig. 6. (Color online) Multi-SLM holographic display setup. (a) Scheme, and (b) photo.)

normal to the illumination direction [13]. This design results in an increased size of angular gap in the display arrangement and with a limited number of SLMs in the design. The display design presented in this paper is much simpler. Since all of the SLMs are illuminated with parallel beams we can pack the SLMs on the circle as closely as possible minimizing angular gaps. Moreover, we can simply reconfigure our display for different reconstruction distances; this is complicated in the previously reported design. One of the main features of our design is the decoupling of the capture and display systems. In between the capture and display stages we have to introduce a processing stage. The display system cannot replay holograms directly as provided by the capture system. In the capture system the CCD is normal to the object center while this is not the case in the display. We have to propagate the optical field from the plane normal (CCD plane) to the tilted plane (SLM plane). We have developed a processing algorithm that couples both systems [16]. Based on a rigorous propagation algorithm between rotated planes it propagates an optical field from the CCD plane to the tilted SLM plane, without an unnecessary increase of resolution.

We have to mention here that the capture and display systems employ different sampling and wavelength parameters. This has a minor effect on behavior of our system; it simply introduces transverse $\left(m_{t}\right)$, longitudinal $\left(m_{l}\right)$, and angular magnification $\left(m_{a}\right)$ [13]. In our case these magnifications are: $m_{t}=1.08 \overline{11}, m_{l}=1.7248$, and $m_{a}=0.6268$.

In order to provide high quality reconstructions the LCoS SLMs have to be calibrated. We have to calibrate both wave aberration [20] and the nonlinearity of the SLM phase response [21]. Each SLM is calibrated individually. Both elements of the calibration are tilt dependent [16]. To calibrate the wave aberration in the tilted SLM configuration we measure the SLM aberration in a normal configuration first and then we recompute this aberration for the tilted SLM geometry. The result is used to remove the aberration effect. The calibration of the nonlinearity is more problematic, as it has to be performed for individual tilt angles. To find the effect of the dependence of the calibration curve on the SLM tilt angle we calibrated a single SLM for a series of tilt angles. We have found that for a SLM tilt of $10 \mathrm{deg}$ the application of a calibration curve for $0 \mathrm{deg}$ gives a phase error below $\lambda / 67$ [16]. Therefore for angles below 10 we can apply a $\overline{0}$ deg calibration curve.

We have configured the display for a reconstruction distance $z_{r}=700 \mathrm{~mm}$. The tilts of the six SLMs for this distance are: $-2.62^{\circ},-1.57^{\circ},-0.52^{\circ}, 0.52^{\circ}$, $1.57^{\circ}, 2.62^{\circ}$ respectively. Therefore, as mentioned above, their effect for SLMs calibration can be neglected i.e., the SLM calibration curve for 0 deg is applied for all modulators. For this display configuration the field of view is $46.5 \mathrm{~mm}$ and the viewing angle 12 deg. According to Eq. (2) the minimum observation distance, when for axial monocular observation $\mathrm{FoV}=\mathrm{MFoV}$, is $z_{\text {olim }}=304 \mathrm{~mm}$. However, as it was discussed in Section 2 for binocular and stereoscopic perception of a holographic image, the viewer must be further away than $z_{\text {olim }}$ and the binocular perception limit distance where both eyes can see reconstructions in the entire $F O V$ area is given by Eq. (3). For the configuration discussed above and taking the average interpupillary distance for females and males $d_{b}=63.8 \mathrm{~mm}$ [22] this limiting distance is $z_{b \text { lim }}=722 \mathrm{~mm}$.

In our display the wave fields generated by individual SLMs are not creating a one true synthetic aperture wavefield. To do this we would need to know the positions of the SLMs and CCDs with accuracy in the order of a fraction of a wavelength (also angularly). 
Therefore, in our multi-SLM display system we do not obtain an increase in resolution, though this is possible in theory [23]. Our system is characterized by the resolution of a single SLM. In our previous publication we have measured the resolution of a holographic display with single LCoS SLM [15]. The practically measured resolution agrees well with the theory. For a reconstruction distance of $700 \mathrm{~mm}$ the theoretical value of resolution is $20.6 \mathrm{~mm}^{-1}$.

\section{Experimental Results}

The functionality of the display system is shown by experiment in two stages: at first it is based on the reconstruction of a set of specially designed synthetic holograms, and secondly, on the reconstruction of digital holograms of real world objects captured in the system described in Section 3. The initial holographic data, calculated or captured, have been numerically modified in order to couple with the display parameters and take into account the SLM tilts with reference to the optical axis. For both cases we use a reconstruction distance of $700 \mathrm{~mm}$.

The synthetic holograms are designed using the method outlined in our previous work [16]. The object is a 3-D model of Gargoyle statue (Fig. ${ }^{7}$ ). The statue was measured with fringe projection system [24] which provides its $3-\mathrm{D}$ mesh representation. Using this 3-D data we have generated several sets of synthetic holograms, each set for different angular orientation of the statue. In Fig. 7 we show the 3-D model in different rotational positions and the corresponding holographic images captured using a digital camera. The vertical regions with lower intensity relate to the gaps that were discussed in Section $\underline{2}$ (Eqs. 5 and 6).

For real world object holograms a scene composed of two small chairs of different sizes was captured in the system described in Section 3. The scene elements were printed using a 3-D printer (ZPrinter 650). The holograms were captured at a distance

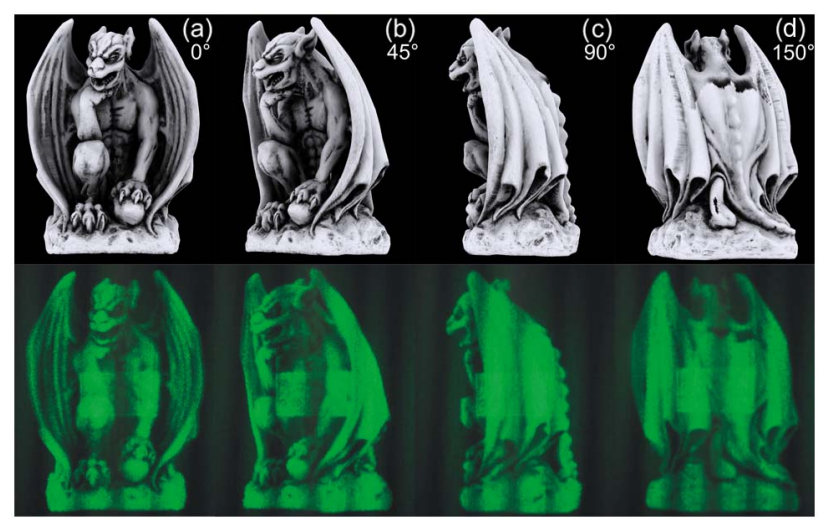

Fig. 7. (Color online) Perspectives of 3-D model of a Gargoyle statue and captured views of holographic images obtained for the statue with different angular orientations. (a) $0^{\circ}$. (b) $45^{\circ}$. (c) $90^{\circ}$. (d) $150^{\circ}$. The size of the front view reconstruction is $33 \times 45 \mathrm{~mm}$ (Media 1). of $406 \mathrm{~mm}$ from the CCD and the reconstruction distance for the display was $700 \mathrm{~mm}\left(m_{l}=1.7248\right)$. As the holographic capture and display systems use coherent illumination, the reconstructed images suffer from significant speckle noise. Reduction of coherence is a frequently applied technique for reducing speckle. However, it limits the space-bandwidth product of the captured signal in both digital holographic recording and holographic displays $[25,26]$. In this paper we therefore use another solution which has been mentioned in Section 3 in which the temporal bandwidth of the SLMs is used for noise reduction. The SLMs have a $60 \mathrm{~Hz}$ refresh rate. In our display we reduce the frame rate by four, and we sequentially display four holograms of the same scene, but with a different speckle pattern thereby reducing the speckle by averaging. In Fig. 8 we show the result of the reconstruction process where all four holograms were displayed in sequence and reconstructed image was captured using a long exposure time with a digital camera. Figure 8(a) shows the images obtained without using the speckle reduction technique while Fig. 8(b) shows the images after speckle reduction. The images are captured for a single SLM reconstruction and different angular orientations of the scene. In order to quantitatively evaluate the reduction in speckle noise we have compared the speckle contrast $C$ for reconstructions presented in Fig. 8(a) and 8(b). The speckle contrast can be computed as a ratio of standard deviation to mean value [18] calculated for a region of uniform distribution of intensity. We have selected regions of constant intensity for chosen reconstructions of the chair object. For these regions we have computed a speckle reduction factor $C_{\text {red }}$ as a ratio of speckle contrast with and without speckle reduction and we have obtained $C_{\text {red }}=0.7$. For the numerical reconstructions shown in Fig. $\underline{5}$ we calculate a speckle reduction factor $C_{\text {red }}=0.52$. This is very close to the theoretical value equal 0.5 .

For the chairs scene the holograms for all six SLMs were captured by rotating the object and then capturing four holograms, each corresponding to a different diffuse illumination. In Fig. 9 we present on axis binocular perception (left and

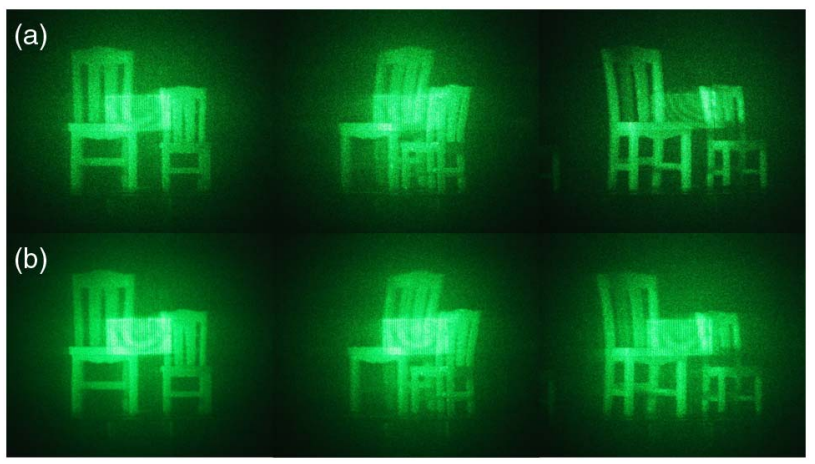

Fig. 8. (Color online) Holographic reconstructions obtained (a) without and (b) with applied noise removal technique. The size of the front view reconstruction is $32 \times 43 \mathrm{~mm}$. 


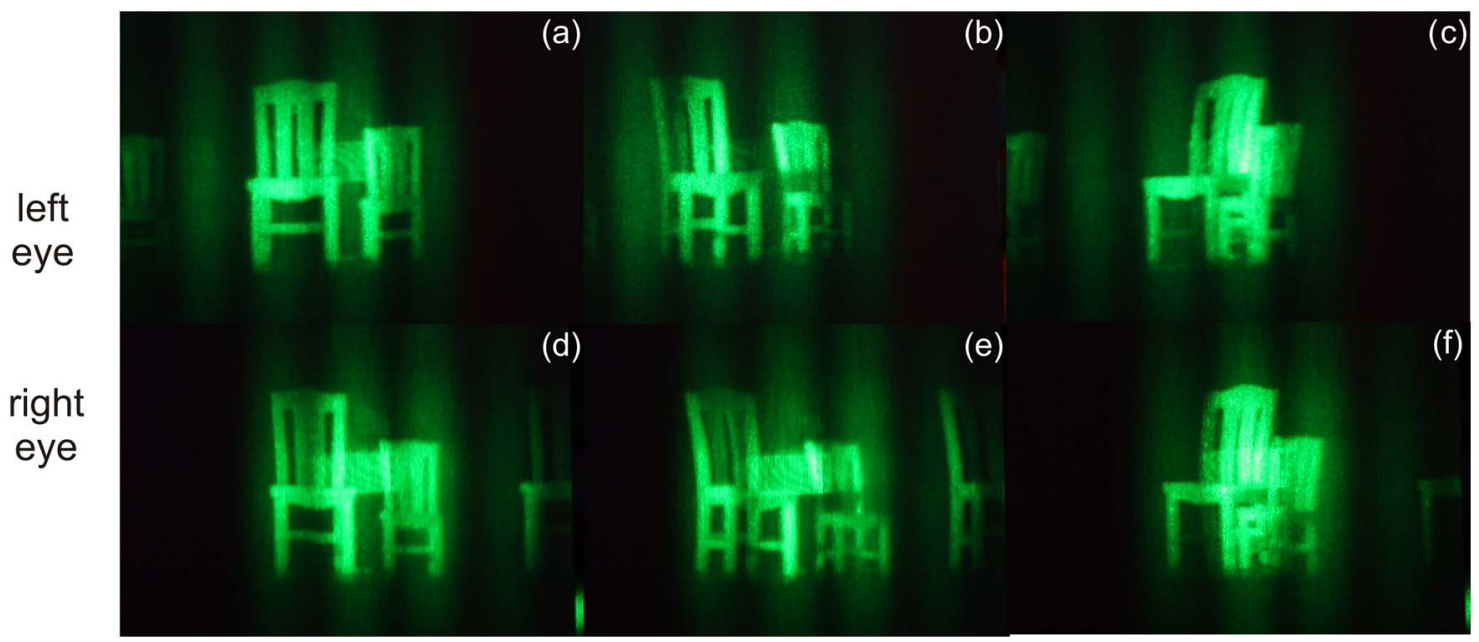

Fig. 9. (Color online) Binocular perception of holographic images captured in 6 SLMs holographic display system. (a), (b), (c) Left eye views. (d), (e), (f) Right eye views captured with a digital camera (Media 2).

right eye views) of the holographic reconstructed image. Left and right eye views were captured with a digital camera placed at observation distances: $\left[z_{o}, x_{o L}\right]=[730 \mathrm{~mm},-32.5 \mathrm{~mm}]$ and $\left[z_{o}, x_{o R}\right]=$ [730 $\mathrm{mm}, 32.5 \mathrm{~mm}]$. The observation distance was chosen according to Eq. (3). To present the views as an eye perceives them we have set the entrance pupil diameter of digital camera to $8.2 \mathrm{~mm}$. This value is close to human observation condition in a dark room (eye aperture diameter $8 \mathrm{~mm}$ ). In Fig. 9(a)-9(c) the left eye views of the reconstructed scene are presented, while in Figs. 9(d)-9(f) right eye views. This proves that in our system we are able to view images stereoscopically (binocularly).

\section{Conclusions}

This paper presents the novel concept of a wide viewing angle holographic display system with multiple tilted SLMs in a circular configuration which allows reconstructions of holograms of both synthetic and 3 -D real world scenes. The main advantage of this display is the capability to decouple the capture and display systems. In the future we believe this will allow for capturing 3-D holographic content using different recording systems, and after numerical processing, replaying it in the standard configuration holographic display.

In the theoretical part of the paper based on a Wigner distribution function analysis we have shown that the parameters describing the ideal holographic display with its pixels arranged circularly is approximately equivalent to the one with circularly distributed planar SLMs (some of the bandwidth is lost). We have shown that this configuration is preferable due to constant resolution over the entire $F_{O} V$ of the image and the optimum usage of the available bandwidth. Also the formulas for a number of important parameters characterizing the conditions for visual perception were derived including: the minimum observation distance at which, for axial observation, $\mathrm{FoV}=\mathrm{MFoV}$; the relation for $M F_{O} V$ as a function of an observer position; and the minimum observation distance for stereoscopic observation. The WDF representation of the real display consisting of six planar SLMs is provided and discussed with respect to the expected effects in the reconstructed image. The effect of gaps between the SLMs is investigated both theoretically and experimentally and we also experimentally validate the necessary conditions that we derive for binocular observation of an image composed of six reconstructions.

The holographic capture system that we use to capture data for the display is based on phase shifting digital holography. In order to simulate the capture of multiple CCDs we use an object located at a rotational table. This configuration allows us to record high quality stop motion videos. However, it cannot provide videos of real world moving scenes. In order to further enhance the quality of the reconstructed images a speckle reduction method which makes use of the temporal bandwidth of SLM was proposed and implemented. It relies on recording holograms of the same scene with multiple (in our case four) different diffuser illumination and later replaying them at our display within the integration time for the human eye. We have presented experiments demonstrating the speckle reduction (Fig. 8).

In summary, the holographic display which has been reported in this paper allows reconstruction of high quality stop motion holographic videos of real world scenes with a size of several centimeters. These scenes were captured by a recording system with parameters that do not match the display ones. It is important to mention that there remain two issues to be considered. The display system is not able to reconstruct a total object field due to the presence of gaps between the SLM and this affects the quality of the reconstructed image, which is disturbing for an observer. Also, there is no coherent stitching of individual wave fields from two SLMs, so we do not create a true synthetic aperture wave field in our display. 
The research leading to these results has received funding from the EU 7th Framework Program FP7/ 2007-2013 under agreement 216105 ('Real 3-D' Project) and the statutory funds of Warsaw University of Technology.

\section{References}

1. F. Yaras, H. Kang, and L. Onural, "State of the art in holographic displays: a survey," J. Display Technol. 6, 443-454 (2010).

2. I. Sexton and Y. Surman, "Stereoscopic and autostereoscopic display systems," Signal Process. Mag. 16, 85-99 (1999).

3. T.-C. Poon, ed., Digital Holography and Three-Dimensional Display (Springer, 2006).

4. N. Hashimoto and S. Morokawa, "Real-time electroholographic system using liquid crystal television spatial light modulators," J Electron. Imaging 2, 93-99 (1993).

5. M. Stanley, M. A. Smith, A. P. Smith, P. J. Watson, S. D. Coomber, C. D. Cameron, and C. W. Slinger, "3-D electronic holography system using a 100 mega-pixel spatial light modulator," Proc. SPIE 5249, 297-308 (2004).

6. R. Haussler, A. Schwerdtner, and N. Leister, "Large holographic display as an alternative to stereoscopic displays," Proc. SPIE 6803, 68030M (2008).

7. A. Michałkiewicz, M. Kujawińska, T. Kozacki, X. Wang, and P. J. Bos, "Holographic three-dimensional displays with liquid crystal on silicon spatial light modulator," Proc. SPIE 5531, 85-94 (2004).

8. D. P. Kelly, D. S. Monaghan, N. Pandey, T. Kozacki, A. Michałkiewicz, G. Finke, B. M. Hennelly, and M. Kujawinska, "Digital holographic capture and optoelectronic reconstruction for 3-D displays," Int. J. Digital Multimedia Broadcasting 2010, 759329 (2010).

9. J. Hahn, H. Kim, Y. Lim, G. Park, and B. Lee, "Wide viewing angle dynamic holographic stereogram with a curved array of spatial light modulators," Opt. Express 16, 12372-12386 (2008).

10. F. Yaras, H. Kang, and L. Onural, "Multi-SLM holographic display system with planar configuration," in Proceedings of IEEE Conference on The True Vision-Capture, Transmission and Display of 3-D Video (IEEE, 2010), pp. 1-4.

11. F. Yaraş, H. Kang, and L. Onural, "Circular holographic video display system,” Opt. Express 19, 9147-9156 (2011).
12. G. Finke, T. Kozacki, and M. Kujawinska, "Wide viewing angle holographic display with multi spatial light modulator array," Proc. SPIE 7723, 77230A (2010).

13. T. Kozacki, M. Kujawinska, G. Finke, W. Zaperty, and B. Hennelly, "Wide viewing angle holographic capture and display systems in circular configuration," J. Display Technol. (to be published)

14. M. Testorf, B. M. Hennelly, and J. O. Castenada, Phase Space Optics (McGraw-Hill, 2009).

15. T. Kozacki, "On resolution and viewing of holographic image generated by 3-D holographic display," Opt. Express 18 27118-27129 (2010).

16. T. Kozacki, "Holographic display with tilted spatial light modulator," Appl. Opt. 50, 3579-3588 (2011).

17. I. Yamaguchi and T. Zhang, "Phase-shifting digital holography," Opt. Lett. 22, 1268 (1997).

18. J. W. Goodman, Speckle Phenomena in Optics (Roberts, 2007).

19. B. M. Hennelly and J. T. Sheridan, "Generalizing, optimizing, and inventing numerical algorithms for the fractional Fourier, Fresnel, and linear canonical transforms," J. Opt. Soc. Am. A 22, 917-927 (2005).

20. A. Bergeron, J. Gauvin, F. Gagnon, D. Gingras, H. H. Arsenault, and M. Doucet, "Phase calibration and applications of a liquid-crystal spatial light modulator," Appl. Opt. 34, 5133-5139 (1995).

21. X. Xun and R. W. Cohn, "Phase calibration of spatially nonuniform spatial light modulators," Appl. Opt. 43, 6400-6406 (2004).

22. H. Gross, F. Blechinger, and B. Achtner, Handbook of Optical Systems, volume 4: Survey on Optical Instruments (Wiley, 2008).

23. S. B. Hasan and T. Kozacki, "Method for enhancing the resolution of holographic displays," Photon. Lett. Poland 1, 193-195 (2009).

24. R. Sitnik, M. Kujawińska, and J. Woźnicki, "Digital fringe projection system for large-volume $360 \mathrm{deg}$ shape measurement," Opt. Eng. 41, 443-449 (2002).

25. T. Kozacki and R. Jóźwicki, "Image reconstruction in near field digital holography with partially coherent illumination," Opt. Commun. 252, 188-201 (2005).

26. F. Yaraş, H. Kang, and L. Onural, "Real-time phase-only color holographic video display system using LED illumination," Appl. Opt. 48, H48-H53 (2009). 